\title{
TRAP1 revisited: Novel localizations and functions of a 'next-generation' biomarker (Review)
}

\author{
MARIA ROSARIA AMOROSO ${ }^{1,2^{*}}$, DANILO SWANN MATASSA $^{2 *}$, LORENZA SISINNI $^{3}$, \\ GIACOMO LETTINI $^{3}$, MATTEO LANDRISCINA ${ }^{4}$ and FRANCA ESPOSITO ${ }^{2}$
}

\author{
${ }^{1}$ Department of Life Sciences, University of Modena and Reggio Emilia, Modena; ${ }^{2}$ Department of Molecular Medicine and \\ Medical Biotechnology, University of Naples Federico II, Naples; ${ }^{3}$ Laboratory of Pre-Clinical and Translational Research, \\ IRCCS, Referral Cancer Centre of Basilicata, Rionero in Vulture, PZ; ${ }^{4}$ Clinical Oncology Unit, \\ Department of Medical and Surgical Sciences, University of Foggia, Foggia, Italy
}

Received April 15, 2014; Accepted May 26, 2014

DOI: $10.3892 /$ ijo.2014.2530

\begin{abstract}
In the last decade, the identification and characterization of novel molecular mechanisms and pathways involving the heat shock protein TRAP1/HSP75 in cancers and other diseases enhanced the scientific interest. Recent reports have shown that TRAP1 stays at the crossroad of multiple crucial processes in the onset of neoplastic transformation. In fact, TRAP1: i) contributes to the tumor's switch to aerobic glycolysis through the inhibition of succinate dehydrogenase, the complex II of the mitochondrial respiratory chain; ii) is part of a pro-survival signaling pathway aimed at evading the toxic effects of oxidants and anticancer drugs and protects mitochondria against damaging stimuli via a decrease of ROS generation; iii) controls protein homeostasis through a direct involvement in the regulation of protein synthesis and protein co-translational degradation. Therefore, TRAP1 seems
\end{abstract}

Correspondence to: Professor Franca Esposito, Department of Molecular Medicine and Medical Biotechnology, University of Naples Federico II, Via S. Pansini 5, I-80131 Naples, Italy

E-mail: franca.esposito@unina.it

Dr Matteo Landriscina, Department of Medical and Surgical Sciences, University of Foggia, Viale Pinto 1, I-71100 Foggia, Italy

E-mail: matteo.landriscina@unifg.it

*Contributed equally

Abbreviations: CAKUT, congenital anomalies of the kidney and urinary tract; CRC, colorectal carcinoma; CypD, cyclophilin D; ER, endoplasmic reticulum; ETC, electron transport chain; HSP, heat shock protein; KO, knock-out; KD, knock-down; MTP, mitochondrial transition pore; OCR, oxygen consumption rate; PD, Parkinson's disease; PTP, permeability transition pore; TRAP1, tumor necrosis factor receptor-associated protein 1; VACTERL, vertebral anomalies, anal atresia, cardiovascular anomalies, tracheoesophageal fistula, renal and/or radial anomalies, limb defects; OS, oxidative stress

Key words: TRAP1, tumor biomarker, apoptosis, anticancer agents, heat shock proteins, protein quality control to be a central regulatory protein with balancing functions at the intersection of different metabolic processes during the neoplastic transformation. For this reason, it can be considered at the same time an attractive target for the development of novel anticancer strategies and a promising study model to understand the biology of tumor cells at a systemic level. This review summarizes the most recent advances in TRAP1 biology and proposes a new comprehensive view of its functions.

\section{Contents}

1. TRAP1 milestones

2. TRAP1 in cancers

3. TRAP1 as an oncogene?

4. TRAP1 as a tumor suppressor?

5. TRAP1 outside the mitochondria: quality control of mitochondrial proteins

6. TRAP1 in health and disease

7. Concluding remarks and future perspectives

\section{TRAP1 milestones}

Although twenty years have passed since TRAP1/HSP75 was firstly identified, only during recent years some light has been shed on its molecular functions. The cloning of tumor necrosis factor receptor-associated protein 1 (TRAP1) as a type I tumor necrosis factor receptor-associated protein, and the identification of HSP75 as a retinoblastoma protein $(\mathrm{Rb})$-binding protein, were independently performed by two different groups, and it was immediately clear that they had identified the same protein $(1,2)$. TRAP1 belongs to the HSP90 chaperone family (3), sharing $26 \%$ identity and $45 \%$ similarity with cytosolic HSP90 (4). Bioinformatic analysis and microscopic observations suggest that TRAP1 is mostly localized to mitochondria and is targeted to the organelle by its $\mathrm{N}$-terminal presequence (5). Interestingly, the ATPase activity of TRAP1 is inhibited by both geldanamycin and radicicol, which have been shown to block specifically HSP90 function; however, 
in vitro experiments showed that TRAP1 does not bind and fold HSP90 client proteins, suggesting distinct functional properties. Quantitative immunogold electron microscopy and biochemical analysis confirmed the mitochondrial distribution of TRAP1 in rat tissues, but additionally revealed a number of non-mitochondrial locations, including nuclei (6).

Following its identification, TRAP1 attracted increasing interest for its homology to HSP90, which has long been pursued for novel cancer therapeutics, coupled with a distinct subcellular localization. Consequently, the majority of the studies focused on expression of this protein in tumors: immunohistochemical staining revealed that TRAP1 is strongly expressed in tumor cells of adenocarcinomas of pancreas, breast, colon, and lung. Conversely, normal matched epithelia contain very low levels of TRAP1 (7). We strongly contributed to these studies, demonstrating that TRAP1 expression is upregulated in approximately $60 \%$ of human colorectal cancers and correlates with a multi-drug resistant phenotype in colon carcinoma cells (8).

Our group was among the first to demonstrate TRAP1 involvement in stress-adaptive response of cancer cells: high levels of both TRAP1 mRNA and protein were found in Saos-2 osteosarcoma cells chronically adapted to mild oxidative conditions (9). Even more interestingly, we identified TRAP1 as a key target in the previously hypothesized correlations between resistance to antitumor agents and adaptation to oxidative stress (OS), since very high levels of this protein were analogously found in tumor cells resistant to 5-fluorouracil and to platin derivatives. However, the most striking data came from the observation that TRAP1 interference, as well as the use of dominant negative mutants of TRAP1, sensitized OS/ chemoresistant cells to cell death inducers, thus supporting the hypothesis of common mechanisms shared by chemoresistance and adaptation to OS and providing the first evidence that TRAP1 is an important player in the development and the maintenance of these phenotypes. Indeed, TRAP1 hyperexpressing cells show a decreased cleavage of the apoptotic markers Caspase 3 and PARP, and increased levels of the scavenging tripeptide GSH. Hence, TRAP1 may be considered a reliable tool to investigate the correlations between oxidative stress, resistance to apoptosis and chemoresistance $(8,9)$.

A role of TRAP1 in the protection from apoptosis and its consequent involvement in the onset and maintenance of tumor phenotypes was firstly and elegantly described by Kang et al (7). These authors discovered that only tumor cells organize a mitochondrial chaperone network, which involves HSP90, its homolog TRAP1 and the immunophilin cyclophilin D (CypD) in a physical complex that regulates permeability transition pore (PTP) opening, maintaining mitochondrial homeostasis and antagonizing the function of CypD in permeability transition. Considering the high 'druggability' of HSP90 ATPase pocket, Kang and colleagues developed a mitochondriadirected variant of 17-AAG carrying the Antennapedia peptide (called Shepherdin) that efficiently accumulates inside the mitochondria, binds mitochondrial TRAP1 and HSP90, and inhibits their chaperone activity via an ATP competition mechanism, thus resulting in CypD-mediated cell death. These observations labelled TRAP1 as an essential controller of mitochondrial homeostasis in tumor cells, conferring them resistance to apoptosis and a survival advantage over the normal counterpart (7). The cytoprotective effect of TRAP1 was further investigated by our group through the identification and functional characterization of TRAP1 interaction with the novel mitochondrial isoform of Sorcin, this providing the first demonstration of a new antiapoptotic complex (10).

TRAP1 role in cell death protection, beyond the direct control of PTP opening in concert with HSP90 and CypD even further characterized by other studies $(11,12)$, may involve some other more general homeostatic mechanisms, linking the control of proteostasis inside mitochondria to the activation of extramitochondrial survival pathways. In this context, we have shown that TRAP1 function could be relevant in crosstalk between mitochondria and other subcellular compartments (3). Accordingly, the HSP90 mitochondrial network contributes to the folding of mitochondrial proteins; however, when this process is dysregulated, a series of cytoprotective/adaptive cellular responses occurs, including activation of autophagy, inter-organelle stress response and induction of gene expression modifications, that lead to the impairment of tumor cell bioenergetics (13). These processes may further increase the apoptotic threshold in tumor cells undergoing mitochondriotoxic stress, ending up with better survival. This adaptive model is highly suitable to enhance the protein buffering capacity of transformed cells, which are especially at risk of proteotoxic stress for their high biosynthetic requirements.

\section{TRAP1 in cancers}

In previous years, substantial data from several groups confirmed the involvement of TRAP1 in tumor biology. A general overview supports the role of TRAP1 in a wide range of cancer types: Zhang et al (14) analyzed the effect of polyphenols of green tea extract (GTE), which exhibit multiple antitumor activities in various cancers, on pancreatic ductal adenocarcinoma and found that GTE inhibited molecular chaperones HSP90, TRAP1 and HSP27 concomitantly in HPAF-II cells. In colorectal carcinomas (CRC) Gao et al (15) found that the increase in TRAP1 expression level was significantly correlated to increased lymph node metastases, advanced tumor stage and reduced overall survival. This suggests that TRAP1 plays an important role in the progression of CRC from a localized to a locoregional metastatic disease and makes TRAP1 a candidate biomarker, predictive for the poor outcome of CRC patients. Consistently, Han et al (16) found that, in patients with metastatic CRC who received first-line oxaliplatin/5-fluorouracil therapy, lower TRAP1 levels correlated with increased overall survival. Furthermore, involvement of TRAP1 in neuroblastoma was recently suggested by Zhan et al (17). Interestingly, TRAP1 was found to be upregulated in hepatocellular carcinoma (HCC) (18), thus the chaperone could have the potential to act as diagnostic HCC biomarker candidate. Analogously, Im and Seo (19) showed that TRAP1 is highly expressed and the mitochondrial mass is decreased in lung carcinoma cell line A549 compared with a normal lung fibroblast. TRAP1 knockdown also reduces cell growth and clonogenic cell survival in non-small cell lung cancer (NSCLC) cells, impairing ATP production and mitochondrial membrane potential. Accordingly, immunohistochemical analysis, performed to evaluate the prognostic potential of TRAP1 expression in NSCLCs, revealed that 
high TRAP1 expression was associated with increased risk of disease recurrence (20).

Altogether, these data further support the involvement of TRAP1 in biogenesis and progression of several kinds of tumors. Most cancers exhibit an antiapoptotic threshold higher than controls, which contributes to disease progression. Hyperexpression of TRAP1 could contribute to this phenotype, thus assigning to this chaperone a leading role in malignancies and an increasing value as a biomarker. The significance of TRAP1 hyperexpression in tumor cells is provided by the evidence that the mitochondrial pool of HSP90s has evolved to face stress conditions and to maintain mitochondrial proteostasis and energetic balance almost exclusively in tumor cells, thus providing a survival advantage. This issue has been recently addressed by Chae et al (21): a proteomic analysis, comparing cells treated with the mitochondrial inhibitor of HSP90s (Gamitrinib) with control cells, showed a modulation in the expression levels of proteins involved in essential mitochondrial processes, such as ribosomal proteins, components of mitochondrial translation apparatus, regulators of purine biosynthesis and the methyl cycle, effectors of oxidative phosphorylation, thus leading to global defects in tumor cell metabolism. Gamitrinib-treated cells, as far as TRAP1 KD cells, exhibited aberrant accumulation of citric acid cycle metabolites, such as succinate, fumarate and malate, and loss of ATP production. A deeper analysis showed that TRAP1 binds the electron transport chain complex II subunit succinate dehydrogenase-B (SDHB), whose solubility is highly decreased in tumor cells upon treatment with Gamitrinib. Accordingly, it was shown that mitochondrial chaperones' function is crucial in maintaining cellular respiration under low-nutrient conditions, protecting complex II in conditions of oxidative stress, and contributing to hypoxia-inducible factor- $1 \alpha$ (HIF-1 $\alpha$ )mediated tumorigenesis in patients carrying SDHB mutations. This pathway may be ideally suited to buffer the risk of proteotoxic stress in transformed cells with high biosynthetic need, preserve organelle integrity against CypD-dependent apoptosis and maintain multiple sources of energy production, especially under stress conditions, such as hypoxia and nutrient deprivation. Thus, HSP90-directed proteostasis in mitochondria regulates tumor cell metabolism, and is gaining increasing importance as a mechanistic explanation for cancer homeostatic regulation, potentially contributing to disease maintenance.

\section{TRAP1 as an oncogene?}

Other outstanding breakthroughs in TRAP1 biology come from evidence by Sciacovelli et al (22): this elegant recent study provides the first demonstration that TRAP1 may behave as an oncogene able to promote neoplastic transformation in different cell systems. The most striking data demonstrated that TRAP1 knock-down (KD) by RNA interference abrogates the transforming potential of different cancer cell lines in in vitro focus forming and soft agar assays, as well as after cell injection in nude mice. Conversely, overexpression of TRAP1 in non-transformed fibroblasts led to cell transformation in analogous assays. Interestingly, overexpression of a TRAP1 deletion mutant, unable to localize into mitochondria, was ineffective, thus suggesting that TRAP1 transforming potential is due to its mitochondrial localization/function. Remarkably, these authors demonstrated that TRAP1 controls mitochondrial metabolism, contributing to the Warburg phenotype. Accordingly, data showed that TRAP1 directly binds complex II and IV of the electron transport chain and inhibits succinate dehydrogenase (SDH) activity in Saos-2 osteosarcoma cells, without affecting the cytochrome oxidase enzymatic activity of complex IV, complex II protein levels or mitochondrial mass. The use of the TRAP1 inhibitor 17AAG in isolated mitochondria restored SDH activity, independently of HSP90. These results were supported by evidence that TRAP1 expression and SDH activity are inversely correlated in colon cancer specimens previously characterized for higher levels of TRAP1 expression compared to surrounding healthy mucosa (8). Strikingly, TRAP1 downregulation markedly increased mitochondrial-dependent respiration in Saos-2 cells in oxygen consumption rate (OCR) experiments, whereas a high proportion of the intracellular ATP content in control cells is provided by glycolysis. Symmetrically, TRAP1 expression in non-transformed fibroblast reduced mitochondrial respiration, thus mimicking the respiratory pattern of cancer cells. It is known that succinate contributes to HIF1 $\alpha$ stability through inhibition of prolyl-hydroxylases (PHDs), whose activity leads to HIF1 $\alpha$ ubiquitin-dependent degradation; therefore, these authors analyzed HIF1 $\alpha$ expression levels during the focus forming assay and found that only TRAP1expressing cells accumulated succinate during the assay, and consequently that HIF $1 \alpha$ was exclusively detectable in this cell population. Consistently, HIF1 $\alpha$ was clearly detected in the majority of cells from tumor samples obtained from nude mice xenografted with TRAP1-expressing Saos-2 cells, whereas HIF1 $\alpha$ downregulation fully abolished the formation of foci in TRAP1-expressing tumor cells and in MEFs transfected with a TRAP1 cDNA. Taken together, the authors propose that TRAP1 induces neoplastic growth through a succinatedependent stabilization of HIF1 $\alpha$. This novel and fascinating hypothesis is schematically summarized in Fig. 1.

\section{TRAP1 as a tumor suppressor?}

Consistently with the observation by Sciacovelli et al (22), Yoshida et al (23) showed that TRAP1 downregulates mitochondrial respiration and ATP production. TRAP1 KO mouse adult fibroblasts (MAFs) displayed a higher basal OCR and a significantly higher maximum respiratory capacity than wildtype (WT) cells, accompanied by decreased glycolysis. This effect was TRAP1-dependent, since re-introduction of TRAP1 in MAFs was sufficient to restore basal OCR levels, confirming a preference for oxidative phosphorylation over aerobic glycolysis in TRAP1 KO cells. In agreement, TRAP1 KO cells showed reduced levels of glycolytic metabolites, increased levels of TCA cycle intermediates and anaplerotic substrates, and increased fatty acid oxidation and $\mathrm{NAD}^{+} / \mathrm{NADH}$ ratio, confirming a metabolic flux through the TCA cycle independent of glucose metabolism. Increased complex IV enzymatic activity was found in TRAP1 KO cells as well as steady-state ATP levels that was significantly higher than in WT cells. These results were confirmed in HeLa and HCT116 human cancer cell lines. In agreement with previous reports, TRAP1dependent inhibition of mitochondrial respiration resulted in 


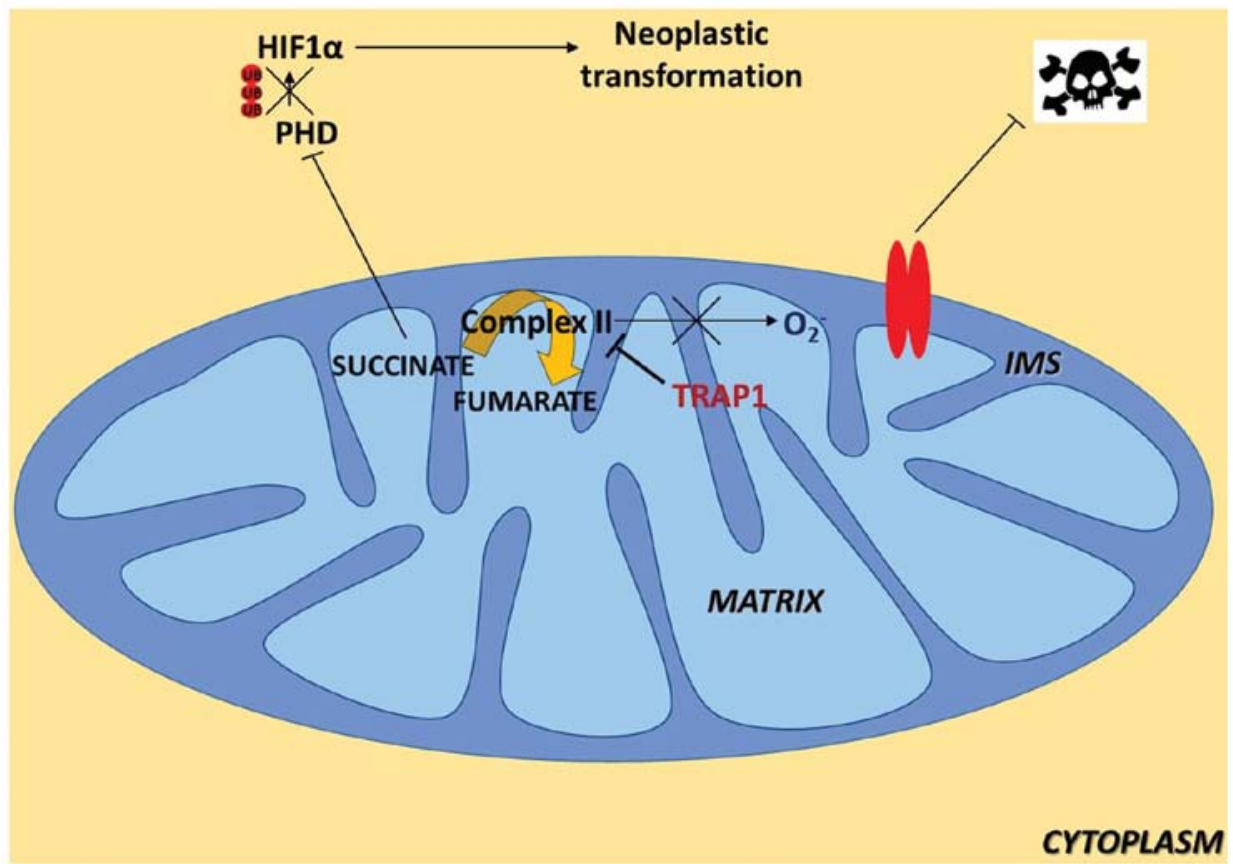

Figure 1. TRAP1 as an oncogene? The respiratory downregulation elicited by TRAP1 interaction with SDH promotes tumorigenesis by priming the succinatedependent stabilization of the pro-neoplastic transcription factor HIF1 $\alpha$ independently of hypoxic conditions.

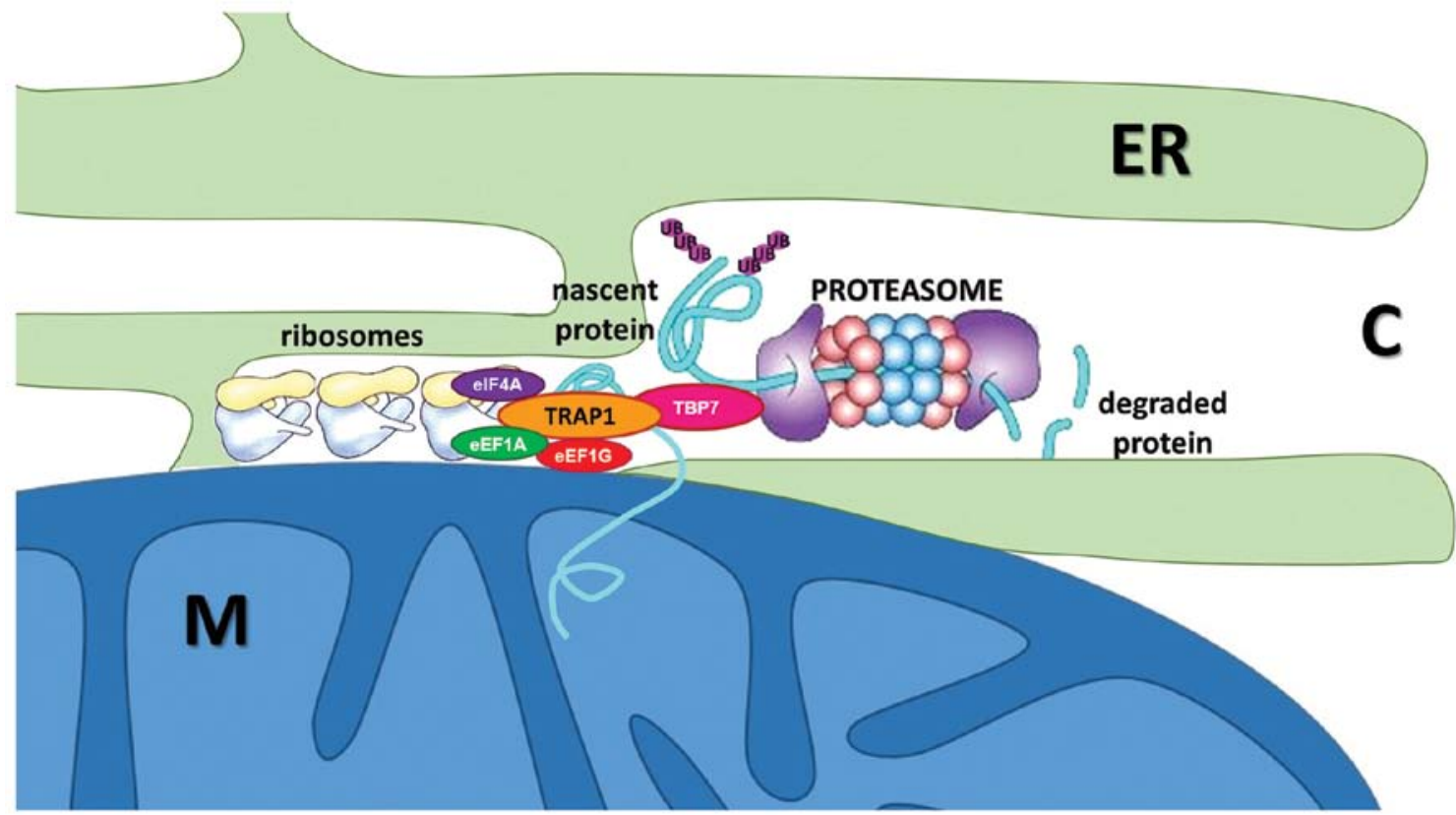

Figure 2. TRAP1 is involved in protein homeostasis. TRAP1 regulates protein expression by coupling synthesis and ubiquitin-dependent degradation, through a direct binding to both ribosomes and the proteasomal particle TBP7, at ER-mitochondria interface. ER, endoplasmic reticulum; M, mitochondrion; C, cytosol.

reduced ROS levels in both MAFs and HCT116, which proves that TRAP1 KO cells are constitutively exposed to elevated oxidative stress. Since elevated ROS favor cell invasion, the authors showed that indeed TRAP1 KO or transient suppression dramatically enhance cell invasiveness, both in mouse fibroblasts and in a variety of human cell lines. Importantly, this phenotype is sensitive to c-Src inhibition and ROS scavenging, supporting a direct link between TRAP1 deficiency, elevated ROS, and c-Src activation in mediating this process. These authors postulated that the impact of TRAP1 on mitochondrial respiration is mediated, at least in part, by its direct regulation of mitochondrial c-Src. In fact, a reciprocal regulation of the two proteins was observed, which led to increased Tyr-416 phosphorylation of mitochondrial c-Src upon TRAP1 suppression, paired with a preferential interaction of TRAP1 with the inactive form of c-Src. 
The impact of reduced TRAP1 expression on in vitro cell invasion led Yoshida and colleagues to hypothesize, contrary to other previously presented findings, that certain more aggressive, metastatic, or late-stage cancer types may express lower TRAP1 levels than less advanced tumors. In particular, they confirmed an inverse correlation between TRAP1 expression and tumor stage in cervical, bladder, and clear cell renal cell carcinoma. Intriguingly, cervical carcinoma is among those cancers whose predominant energy metabolism is obtained via oxidative phosphorylation, not glycolysis. Therefore, the authors suggested a re-evaluation of the assumption that TRAP1 is uniformly pro-oncogenic, and to consider that TRAP1 inhibition alone is unlikely to be a viable treatment strategy for cancers that use oxidative phosphorylation.

The intriguing hypothesis that, in some settings and depending on the cell type context, TRAP1 may act as a tumor suppressor is partially supported by a study on 208 patients affected by ovarian cancer. Immunohistochemical analyses showed that high TRAP1 expression correlated significantly with favorable chemotherapy-response and showed a significantly positive impact on overall survival (24). The authors also found that high TRAP1 expression correlated significantly with estrogen receptor $\alpha$ levels. Consistently, the expression of TRAP1 was previously shown as significantly increased in letrozole-responsive cancers compared to letrozole-insensitive (25). Considering that TRAP1 has been reported as an estrogen-regulated gene (26), it will be interesting, in future studies, to analyze the relationship between TRAP1 and tumor outcome in gynaecological malignancies.

\section{TRAP1 outside the mitochondria: quality control of mitochondrial proteins}

Although original reports already suggested evidence of extramitochondrial localizations of TRAP1, for a long time this has been substantially ignored by the scientific community and TRAP1 has been only considered 'the mitochondrial HSP90', whose role was restricted to the maintenance of organelle homeostasis through the regulation of the mitochondrial transition pore (MTP) as a scavenger of mitochondrial ROS. However, our group started few years ago to 'look' outside the mitochondria: taking advantage of proteomic profiles, mass spectra data, as well as biochemical and microscopic observations, we provided the first demonstration of an extramitochondrial localization of TRAP1 linked to a specific function. This novel and interesting field to explore allowed us to identify a novel interaction of TRAP1 with the proteasomal particle TBP7 on the outer side of the endoplasmic reticulum (ER), whose function controls the fate of two mitochondrial proteins, Sorcin isoform B and F1ATPase $\beta$ subunit, through the regulation of their ubiquitination (27). However, even though the structural characterization of TRAP1/TBP7 interaction and their functional role in the quality control of mitochondria destined proteins was deeply explored in our studies, a question remained unanswered: how can TRAP1 take care of mitochondrial proteins even before they reach their destination? Excluding the hypothesis of a folding control, which is known to occur inside mitochondria, and after the demonstration that TRAP1 does not influence the half-life of substrates (28), we hypothesized a role in the quality control at early steps of protein synthesis. It is now accepted that up to $30 \%$ of neo-synthesized proteins undergo ubiquitination to be cotranslationally degraded if they are damaged (29). Very recent reports support this hypothesis: many groups demonstrated the presence of chaperone complexes and ubiquitination machinery that regulate the first steps of protein synthesis, to ensure that damaged polypeptides are immediately degraded before the complete folding, in many cases while they are still bound to ribosomes (30). These observations open a new scenario in which ribosomeassociated chaperones act as key regulators of cellular proteostasis through direct or indirect modulation of protein synthesis, folding, assembly and transport. Consistently, we found TRAP1 associated to actively translating polysomes and to three translation factors, namely eIF4A, eEF1A, eEF1G (28) in HCT116 CRC cells. This suggests novel roles for TRAP1 in translational processes, which explains the mechanism of quality control on its mitochondrial substrates. At early stages of protein synthesis, TRAP1 substrates are rapidly accumulated but more rapidly degraded in TRAP1 KO cells, indicating that this chaperone regulates the fate of its target proteins through a cotranslational mechanism, controlling the balance between synthesis and degradation. TRAP1 control involves the ubiquitin-proteasome system (UPS): TRAP1 silencing causes an increase in total ubiquitination levels, and this phenotype is rescued by the transfection of an extramitochondrial TRAP1 mutant (27). Consistently, TRAP1 regulation of ubiquitination occurs during translation: recovery of total ubiquitinated proteins by immunoprecipitation, following a brief pulse with radiolabelled amino acids, showed that TRAP1-stable interfered CRC cells accumulate more than double amount of ubiquitinated proteins during protein synthesis. TRAP1dependent regulation of protein synthesis/ubiquitination is schematically represented in Fig. 2.

Recently our group unraveled that this regulatory mechanism is quite complex and involves the modulation of phosphorylation levels, either in basal conditions or under stress, of the translation factor eIF $2 \alpha$, whose phosphorylation results in the attenuation of cap-dependent translation, while favoring the IRES-dependent one. TRAP1 slows the rate of protein synthesis through the eIF2 $\alpha$ pathway, favoring the activation of GCN2 and PERK kinases, with consequent phosphorylation of eIF $2 \alpha$ and attenuation of cap-dependent translation. This enhances the synthesis of selective stressresponsive proteins, as the transcription factor ATF4 and its downstream effectors BiP/Grp78 and the cystine antiporter system $\mathrm{xCT}$, thereby providing protection toward ER stress, oxidative damage and nutrient deprivation. Accordingly, TRAP1 silencing sensitizes cells to apoptosis induced by novel antitumoral drugs that inhibit cap-dependent translation, such as Ribavirin or 4EGI-1, and reduces the ability of cells to migrate through the pores of transwell filters in the presence of these drugs (28). The relevance of these findings is supported by evidence that translational control is a crucial component of cancer development and progression, involved in the regulation of both global protein synthesis and translation of selective mRNAs species that promote tumor cell survival, angiogenesis, transformation, invasion and metastasis (31).

Translational control of cancer is multifaceted, involving alterations in translation factor expression levels and activities 
unique to different types of cancers, disease stages and the tumor microenvironment $(32,33)$. This process allows cells to respond swiftly to a changing environment, and becomes very important during tumorigenesis, when cells have to face stress conditions resulting from reduced oxygen and nutrient availability, and have to reprogram their metabolism. We suggest that TRAP1 could be involved in this adaptation process. Indeed, TRAP1 is hyperexpressed in colorectal and breast carcinoma specimens, and it is co-upregulated with members of translational machinery and with stress-responsive chaperones such as BiP/Grp78. In this perspective, the role of TRAP1 becomes relevant in resistance to antiapoptotic agents that involve ER stress activation, condition often found in tumors. We have shown that TRAP1 CRC interfered cells are more sensitive to thapsigargin (an ER-stress inducer), and very recently confirmed these data in a breast cancer cell model, demonstrating that TRAP1 also confers resistance to paclitaxel, a microtubule stabilizing/ER stress inducer agent widely used in breast cancer therapy (34), and to genotoxic agents, as anthracyclins (35). Accordingly, paclitaxel- and anthracyclin-resistant cell lines show higher levels of TRAP1 compared to the non-resistant counterpart. In this context, the extramitochondrial TRAP1 antiapoptotic activity represents a mechanism responsible for the protection against ER stress, ER stress inducers and a variety of chemotherapeutics causing ER stress: indeed, ER-associated TRAP1 regulates mitochondrial apoptosis by controlling the expression of specific client proteins and, among others, Sorcin, thus suggesting that TRAP1 may be a key player in coupling organelle proteostasis, adaptation to stress and cell survival, through the regulation of the mitochondrial apoptotic pathway. These data are further confirmed by the finding that an extramitochondrial TRAP1 mutant is still able to provide resistance against ER stressinduced cell death and to maintain high levels of mitochondrial cytoprotective proteins such as Sorcin. Thus, TRAP1 is emerging as a key regulator of bidirectional crosstalk between ER and mitochondria, and ER-localized TRAP1 is becoming relevant in the balance of cellular homeostasis since it controls protein synthesis, contributes to cell's proper response to stress conditions, and regulates pathways involved in cytoprotection and drug resistance. In such a perspective, cytoprotective activities of both intramitochondrial and extramitochondrial TRAP1 are involved in the resistance to apoptosis of tumor cells and in protection from traditional chemotherapeutics $(8,10,34,35)$. These new findings confirm that TRAP1 network could be an attractive target to develop novel anticancer strategies aimed at inhibiting TRAP1 function in cell compartments other than mitochondria, in order to disrupt protein quality control mechanisms, lower the threshold of MTP opening selectively in cancer cells and revert the drug-resistant phenotype of human malignancies. In such a perspective, further studies are urgently needed to identify specific subsets of human tumors, which are dependent for their survival on this extramitochondrial quality control network and are, thus, suitable for a TRAP1-targeted therapy.

\section{TRAP1 in health and disease}

Beyond the widely described roles of TRAP1 in the etiopathogenesis of a multifactorial disease as cancer, in the past
10 years very sophisticated and focused studies have unveiled an important involvement of this chaperone in the genesis and development of some human diseases. These include several human neurodegenerative diseases, such as Alzheimer's disease, Parkinson's disease (PD) and Huntington's disease, all considered as 'mitochondropathies', as well as other genetic, and not necessarily mitochondrial, kidney and cardiovascular diseases. Due to our limited contribution and knowledge of these studies the most recent achievements will be briefly summarized, and we apologize to all the authors for not giving enough 'space' to their outstanding results.

TRAP1 in the maintenance of mitochondrial integrity. Changes in mitochondrial morphology, which is regulated by continuous fusion and fission to form highly connected networks or fragmented units, may lead to the degradation of mitochondria via autophagy (so-called mitophagy) and often cause neuronal synaptic loss and cell death in several human neurodegenerative diseases. Therefore, cells have developed complex quality control mechanisms to cope with the different challenges constantly imposed on the integrity of mitochondria. Pridgeon et al (36) have indirectly linked TRAP1 to Parkinson's disease, demonstrating that PINK1, a major kinase whose mutations are involved in the development of autosomic recessive forms of PD, is a binding partner of TRAP1, phosphorylates it upon induction of oxidative stress, and that TRAP1 is required for PINK1-mediated protection against oxidative-stress-induced apoptosis.

Takamura et al (37) showed that TRAP1 KD in neuroblastoma cells and glioma cancer cell lines of neuronal derivation induced an abnormal mitochondrial morphology, through a significant decrease in dynamin-related protein 1 (Drp1) and mitochondrial fission factor (Mff), affecting mitochondrial function. These observations confirm a role of TRAP1 in maintaining mitochondrial morphology.

Some other studies performed in Drosophila systems by Zhang et al (38) demonstrated that human TRAP1 is able to rescue phenotypes in PINK1 loss-of-function flies, but has only minor effects on phenotypes of flies deficient of PARKIN, another protein associated with autosomal recessive, early-onset PD. In addition, detrimental effects observed after RNAi-mediated silencing of complex I subunits were rescued by TRAP1 in Drosophila. Remarkably, these studies confirm the metabolic control by TRAP1, albeit in different experimental systems: in fact, the lack of functional mitochondria obviously coincides with reduced levels of complex I subunits, impaired complex I activity and a decline in ATP content. TRAP1 protective effects require its ATP binding properties, as ATP-binding deficient TRAP1 mutants were unable to rescue this activity. Considering that functional ETC causes polarization of the mitochondrial membrane and that depolarization of mitochondria is required to initiate mitophagy, it can be speculated that TRAP1 might negatively regulate mitophagy by maintaining the ETC in a functional state (38).

Accordingly, Costa et al (39) characterized Drosophila TRAP1 null mutants, showing that loss of TRAP1 results in a decrease in mitochondrial function and increased sensitivity to stress. These findings demonstrated that TRAP1 works downstream of PINK1 and in parallel with PARKIN in Drosophila, 
and that enhancing its function may ameliorate mitochondrial dysfunction and rescue neurodegeneration in PD.

Moreover, TRAP1 protein has been identified as a novel modifier of the mitochondrial toxicity induced by [A53T] $\alpha$-Synuclein in fruitfly models of PD. Cell culture experiments further demonstrated that [A53T] $\alpha$-Synuclein directly interferes with a number of mitochondrial functions, including complex I ATP production, mitochondrial fragmentation, and sensitivity to oxidative stress. These effects could be blocked by TRAP1 overexpression (40).

As mitochondrial dysfunction has been previously linked to mutations in several other genes associated with genetic PD, all the described data provide further evidence of a common mitochondrial-centric mechanism of PD pathogenesis, that probably involves TRAP1 function, its interaction with PINK1 and the regulation of ETC function.

The genetics of TRAP1. In last two years, few pioneering studies suggested an involvement of TRAP1 in renal diseases. Fismen et al (41) analyzed the coregulation between TRAP1 and DNaseI in glomerulonephritis. Previous data indicate that TRAP1 and DNaseI genes overlap; recent findings, moreover, show that transformation of mild glomerulonephritis into end-stage disease coincides with shutdown of renal DNaseI expression in mouse models, damaging glomerular basement membranes. Translating the observations obtained in mice to human lupus nephritis, they observed that DNaseI shutdown coincides with TRAP1 overexpression, with a still unclear, but structured molecular mechanism, that could probably involve transcriptional interference, thus providing to TRAP1 a new role in the progression of this disease.

Our group contributed to a partial characterization of a novel role of TRAP1 in kidney abnormalities of genetic origin. Saisawat et al (42) shed new light on the etiology of congenital anomalies of the kidney and urinary tract (CAKUT) and vertebral anomalies, anal atresia, cardiovascular anomalies, tracheoesophageal fistula, renal and/or radial anomalies, limb defects (VACTERL) association, rare pathologies that affect kidney and urinary tract. Recessive mutations of TRAP1 were found by deep sequencing analysis of the genome from affected patients, eliciting some questions about the role of TRAP1 in the genesis of these pathologies and its involvement in embryonic development of the kidney. In normal tissues, TRAP1 was found to be expressed in renal epithelia of developing mouse kidney and in the kidney of adult rats. Likely, the antiapoptotic role of TRAP1 is important in the correct development of the renal apparatus, and its loss of function due to mutations in its functional domains could be disease-causing. It is worth noting that there are six case reports published of individuals with VACTERL association in conjunction with mitochondrial dysfunction, as summarized recently by Siebel and Solomon (43); this could be consistent with the well known role of TRAP1 in control of mitochondrial integrity and metabolism, thus suggesting a possible relationship between TRAP1 inactivation and the pathogenesis of the disease.

From the overall data, it would not be surprising to hypothesize a role for TRAP1 in normal cellular and tissue development. Indeed, it is well known that HSPs are related to development; in addition, HSP genes are shown to be phase- and tissue-specific during embryogenesis. It was found that 19 HSPs of the HSP70, HSP90, and HSP110 families hold different expression and characteristic correlations with the developmental phases in embryonic forelimb tissue of normal mice. Among those, TRAP1 may be considered as a limbdevelopment-related gene (44). Moreover, in vivo experiments showed that TRAP1 expression could be closely associated with normal palate development and cleft palate in mouse embryo, probably through participating in the stress response process and/or the antiapoptotic processes (45).

\section{Concluding remarks and future perspectives}

The interest for HSP90 as a therapeutic target of several human diseases, from neurodegeneration to cancers, is still very high. However, very few data are available on the isoform specificity profiles of the HSP90 inhibitors. The crystal structure of full-length TRAP1, very recently presented (46), will certainly open a new scenario on the: i) selectivity to target only TRAP1 among all the other members of HSP90 family; ii) possibility to design novel, specific TRAP1 inhibitors to be directed either inside or outside mitochondria.

This review aims at presenting an updated view of this important protein mainly in tumor cell pathophysiology. In our opinion, the core result of recent advances in TRAP1 biology lies in two items of evidence: TRAP1 crucially influences the switch from oxidative metabolism to glycolysis, and therefore contributes to tumorigenesis by inhibiting mitochondrial respiration, and simultaneously affects global protein quality control by contributing to protein synthesis regulation in the ER. This is realized through another focused metabolic switch between prevalent cap- to IRES-dependent translation and the balance between protein synthesis and cotranslational degradation.

TRAP1 seems therefore to be a central regulatory protein with homeostatic roles at the crossroad between different kinds of cell functions/metabolism during the transformation process or, possibly, during normal development, as suggested by the novel and emerging evidence on its involvement in the etiology of human pathologies. Strikingly, TRAP1 function in normal cells is almost unexplored. In this context, the recent findings of TRAP1 involvement in mouse and human embryonic development could stimulate an interesting study of the physiological role of this chaperone.

As widely addressed in this review, TRAP1 has long been considered an attractive biomarker and/or target for future development of novel therapeutic strategies. The apparent discrepancies on several data recently obtained by different outstanding scientists, i.e. stabilization/inhibition of the ETC component activity, HIF-mediated tumorigenesis, TRAP1 as a tumor suppressor or TRAP1 as an oncogene, further reinforce the interest in TRAP1 'life'. In addition, the apparently opposite/contrasting function of TRAP1 in ovarian cancers, compared to its very conserved uniform trend in all other tumor types, including colorectal cancer, suggests that further studies are required to shed light on the role of TRAP1 in different types of human cancers.

Undoubtedly, traditional anticancer chemotherapeutics, targeting DNA replication and cell division, have proved to be highly successful in the treatment of some tumors. However, 
they have severe side effects and limitations, above all the development of resistance. Now, a new wave of anticancer agents is emerging, targeting complex multicomponent cellular machineries, including heat shock protein chaperones and the proteasome, which thus interfere with those support systems that are more essential for cancer cells than for normal cells (47).

As one of the 'oldest' TRAP1 study groups, we strongly hope that both the continuously emerging findings on new TRAP1 features together with the reading of the present review on the 'revisited' TRAP1 could contribute to identify this chaperone as an important novel target in future anticancer, and not only, drug development.

\section{Acknowledgements}

This study was supported by the Associazione Italiana per la Ricerca sul Cancro (AIRC) (Grant IG13128 to M.L. and F.E.), by the Italian Ministry of Health (Grant GR-2010-2310057), by POR Campania FSE 2007-2013, Projects CRÈME and STRAIN.

\section{References}

1. Song HY, Dunbar JD, Zhang YX, Guo D and Donner DB: Identification of a protein with homology to hsp90 that binds the type 1 tumor necrosis factor receptor. J Biol Chem 270: 3574-3581, 1995.

2. Chen CF, Chen Y, Dai K, Chen PL, Riley DJ and Lee WH: A new member of the hsp90 family of molecular chaperones interacts with the retinoblastoma protein during mitosis and after heat shock. Mol Cell Biol 16: 4691-4699, 1996.

3. Matassa DS, Amoroso MR, Maddalena F, Landriscina M and Esposito F: New insights into TRAP1 pathway. Am J Cancer Res 2: 235-248, 2012.

4. Chen B, Piel WH, Gui L, Bruford E and Monteiro A: The HSP90 family of genes in the human genome: insights into their divergence and evolution. Genomics 86: 627-637, 2005.

5. Felts SJ, Owen BA, Nguyen P, Trepel J, Donner DB and Toft DO: The hsp90-related protein TRAP1 is a mitochondrial protein with distinct functional properties. J Biol Chem 275: 3305-3312, 2000.

6. Cechetto JD and Gupta RS: Immunoelectron microscopy provides evidence that tumor necrosis factor receptor-associated protein 1 (TRAP-1) is a mitochondrial protein which also localizes at specific extramitochondrial sites. Exp Cell Res 260: 30-39, 2000.

7. Kang BH, Plescia J, Dohi T, Rosa J, Doxsey SJ and Altieri DC: Regulation of tumor cell mitochondrial homeostasis by an organelle-specific Hsp90 chaperone network. Cell 131: 257-270, 2007.

8. Costantino E, Maddalena F, Calise S, Piscazzi A, Tirino V, Fersini A, Ambrosi A, Neri V, Esposito F and Landriscina M: TRAP1, a novel mitochondrial chaperone responsible for multi-drug resistance and protection from apoptotis in human colorectal carcinoma cells. Cancer Lett 279: 39-46, 2009.

9. Montesano Gesualdi N, Chirico G, Pirozzi G, Costantino E, Landriscina $\mathrm{M}$ and Esposito F: Tumor necrosis factor-associated protein 1 (TRAP-1) protects cells from oxidative stress and apoptosis. Stress 10: 342-350, 2007.

10. Landriscina M, Laudiero G, Maddalena F, Amoroso MR, Piscazzi A, Cozzolino F, Monti M, Garbi C, Fersini A, Pucci P and Esposito F: Mitochondrial chaperone Trap1 and the calcium binding protein Sorcin interact and protect cells against apoptosis induced by antiblastic agents. Cancer Res 70: 6577-6586, 2010.

11. Ghosh JC, Siegelin MD, Dohi T and Altieri DC: Heat shock protein 60 regulation of the mitochondrial permeability transition pore in tumor cells. Cancer Res 70: 8988-8993, 2010.

12. Rasola A, Sciacovelli M, Pantic B and Bernardi P: Signal transduction to the permeability transition pore. FEBS Lett 584: 1989-1996, 2010.
13. Altieri DC: Hsp90 regulation of mitochondrial protein folding: from organelle integrity to cellular homeostasis. Cell Mol Life Sci 70: 2463-2472, 2013.

14. Zhang L, Pang E, Loo RR, Rao J, Go VL, Loo JA and Lu QY: Concomitant inhibition of HSP90, its mitochondrial localized homologue TRAP1 and HSP27 by green tea in pancreatic cancer HPAF-II cells. Proteomics 11: 4638-4647, 2011.

15. Gao JY, Song BR, Peng JJ and Lu YM: Correlation between mitochondrial TRAP-1 expression and lymph node metastasis in colorectal cancer. World J Gastroenterol 18: 5965-5971, 2012.

16. Han JJ, Baek SK, Lee JJ, Kim GY, Kim SY and Lee SH: Combination of TRAP1 and ERCC1 expression predicts clinical outcomes in metastatic colorectal cancer treated with oxaliplatin/5-fluorouracil. Cancer Res Treat 46: 55-64, 2014.

17. Zhan Q, Tsai S, Lu Y, Wang C, Kwan Y and Ngai S: RuvBL2 is involved in histone deacetylase inhibitor PCI-24781-induced cell death in SK-N-DZ neuroblastoma cells. PLoS One 8: e71663, 2013.

18. Megger DA, Bracht T, Kohl M, Ahrens M, Naboulsi W, Weber F, Hoffmann AC, Stephan C, Kuhlmann K, Eisenacher M, Schlaak JF, Baba HA, Meyer HE and Sitek B: Proteomic differences between hepatocellular carcinoma and nontumorous liver tissue investigated by a combined gel-based and labelfree quantitative proteomics study. Mol Cell Proteomics 12: 2006-2020, 2013.

19. Im CN and Seo JS: Overexpression of tumor necrosis factor receptor-associated protein 1 (TRAP1), leads to mitochondrial aberrations in mouse fibroblast NIH/3T3 cells. BMB Rep: Dec 1, 2013 (Epub ahead of print). pii: 2469.

20. Agorreta J, Hu J, Liu D, Delia D, Turley H, Ferguson DJ, Iborra F, Pajares MJ, Larrayoz M, Zudaire I, Pio R, Montuenga LM, Harris AL, Gatter K and Pezzella F: TRAP1 regulates proliferation, mitochondrial function and has prognostic significance in NSCLC. Mol Cancer Res: 3 June, 2014 (Epub ahead of print).

21. Chae YC, Angelin A, Lisanti S, Kossenkov AV, Speicher KD, Wang H, Powers JF, Tischler AS, Pacak K, Fliedner S, Michalek RD, Karoly ED, Wallace DC, Languino LR, Speicher DW and Altieri DC: Landscape of the mitochondrial Hsp90 metabolome in tumours. Nat Commun 4: 2139, 2013.

22. Sciacovelli M, Guzzo G, Morello V, Frezza C, Zheng L, Nannini N, Calabrese F, Laudiero G, Esposito F, Landriscina M, Defilippi P, Bernardi P and Rasola A: The mitochondrial chaperone TRAP1 promotes neoplastic growth by inhibiting succinate dehydrogenase. Cell Metab 17: 988-999, 2013.

23. Yoshida S, Tsutsumi S, Muhlebach G, Sourbier C, Lee MJ, Lee S, Vartholomaiou E, Tatokoro M, Beebe K, Miyajima N, Mohney RP, Chen Y, Hasumi H, Xu W, Fukushima H, Nakamura K, Koga F, Kihara K, Trepel J, Picard D and Neckers L: Molecular chaperone TRAP1 regulates a metabolic switch between mitochondrial respiration and aerobic glycolysis. Proc Natl Acad Sci USA 110: E1604-E1612, 2013.

24. Aust S, Bachmayr-Heyda A, Pateisky P, Tong D, Darb-Esfahani S, Denkert C, Chekerov R, Sehouli J, Mahner S, Van Gorp T, Vergote I, Speiser P, Horvat R, Zeillinger R and Pils D: Role of TRAP1 and estrogen receptor alpha in patients with ovarian cancer - a study of the OVCAD consortium. Mol Cancer 11: 69, 2012.

25. Walker G, MacLeod K, Williams AR, Cameron DA, Smyth JF and Langdon SP: Estrogen-regulated gene expression predicts response to endocrine therapy in patients with ovarian cancer. Gynecol Oncol 106: 461-468, 2007

26. O'Donnell AJ, Macleod KG, Burns DJ, Smyth JF and Langdon SP: Estrogen receptor-alpha mediates gene expression changes and growth response in ovarian cancer cells exposed to estrogen. Endocr Relat Cancer 12: 851-866, 2005.

27. Amoroso MR, Matassa DS, Laudiero G, Egorova AV, Polishchuk RS, Maddalena F, Piscazzi A, Paladino S, Sarnataro D, Garbi C, Landriscina $M$ and Esposito F: TRAP1 and the proteasome regulatory particle TBP7/Rpt3 interact in the endoplasmic reticulum and control cellular ubiquitination of specific mitochondrial proteins. Cell Death Differ 19: 592-604, 2012.

28. Matassa DS, Amoroso MR, Agliarulo I, Maddalena F, Sisinni L, Paladino S, Romano S, Romano MF, Sagar V, Loreni F, Landriscina $\mathrm{M}$ and Esposito F: Translational control in the stress adaptive response of cancer cells: a novel role for the heat shock protein TRAP1. Cell Death Dis 4: e851, 2013.

29. Wang F, Durfee LA and Huibregtse JM: A cotranslational ubiquitination pathway for quality control of misfolded proteins. Mol Cell 50: 368-378, 2013. 
30. Brandman O, Stewart-Ornstein J, Wong D, Larson A Williams CC, Li GW, Zhou S, King D, Shen PS, Weibezahn J, Dunn JG, Rouskin S, Inada T, Frost A and Weissman JS: A ribosome-bound quality control complex triggers degradation of nascent peptides and signals translation stress. Cell 151: 1042-1054, 2012.

31. Ruggero D: Translational control in cancer etiology. Cold Spring Harb Perspect Biol 5: pii a012336, 2013.

32. Silvera D, Formenti SC and Schneider RJ: Translational control in cancer. Nat Rev Cancer 10: 254-266, 2010.

33. Liu B,Han Y and Qian SB: Cotranslational response to proteotoxic stress by elongation pausing of ribosomes. Mol Cell 49: 453-463, 2013.

34. Maddalena F, Sisinni L, Lettini G, Condelli V, Matassa DS, Piscazzi A, Amoroso MR, La Torre G, Esposito F and Landriscina M: Resistance to paclitxel in breast carcinoma cells requires a quality control of mitochondrial antiapoptotic proteins by TRAP1. Mol Oncol 7: 895-906, 2013.

35. Sisinni L, Maddalena F, Lettini G, Condelli V, Matassa DS, Esposito F and Landriscina M: TRAP1 role in endoplasmic reticulum stress protection favors resistance to anthracyclins in breast carcinoma cells. Int J Oncol 44: 573-582, 2014.

36. Pridgeon JW, Olzmann JA, Chin LS and Li L: PINK1 protects against oxidative stress by phosphorylating mitochondrial chaperone TRAP1. PLoS Biol 5: e172, 2007.

37. Takamura H, Koyama Y, Matsuzaki S, Yamada K, Hattori T, Miyata S, Takemoto K, Tohyama M and Katayama T: TRAP1 controls mitochondrial fusion/fission balance through Drp1 and Mff expression. PLoS One 7: e51912, 2012.

38. Zhang L, Karsten P, Hamm S, Pogson JH, Müller-Rischart AK, Exner N, Haass C, Whitworth AJ, Winklhofer KF, Schulz JB and Voigt A: TRAP1 rescues PINK1 loss-of-function phenotypes. Hum Mol Genet 22: 2829-2841, 2013.

39. Costa AC, Loh SH and Martins LM: Drosophila Trap1 protects against mitochondrial dysfunction in a PINK1/parkin model of Parkinson's disease. Cell Death Dis 4: e467, 2013.

40. Butler EK, Voigt A, Lutz AK, Toegel JP, Gerhardt E, Karsten P, Falkenburger B, Reinartz A, Winklhofer KF and Schulz JB: The mitochondrial chaperone protein TRAP1 mitigates $\alpha$-Synuclein toxicity. PLoS Genet 8: e1002488, 2012.
41. Fismen S, Thiyagarajan D, Seredkina N, Nielsen H, Jacobsen S, Elung-Jensen T, Kamper AL, Johansen SD, Mortensen ES and Rekvig OP: Impact of the tumor necrosis factor receptorassociated protein 1 (Trap1) on renal DNaseI shutdown and on progression of murine and human lupus nephritis. Am J Pathol 182: 688-700, 2013

42. Saisawat P, Kohl S, Hilger AC, Hwang DY, Yung Gee H, Dworschak GC, Tasic V, Pennimpede T, Natarajan S, Sperry E, Matassa DS, Stajić N, Bogdanovic R, de Blaauw I, Marcelis CL, Wijers CH, Bartels E, Schmiedeke E, Schmidt D, Märzheuser S, Grasshoff-Derr S, Holland-Cunz S, Ludwig M, Nöthen MM, Draaken M, Brosens E, Heij H, Tibboel D, Herrmann BG, Solomon BD, de Klein A, van Rooij IA, Esposito F, Reutter HM and Hildebrandt F: Whole-exome resequencing reveals recessive mutations in TRAP1 in individuals with CAKUT and VACTERL association. Kidney Int 85: 1310-1317, 2014.

43. Siebel S and Solomon BD: Mitochondrial factors and VACTERL association-related congenital malformations. Mol Syndromol 4: 63-73, 2013.

44. Zhu Y, Zhou H, Zhu Y, Wan X, Zhu J and Zhang T: Gene expression of Hsp70, Hsp90, and Hsp110 families in normal and abnormal embryonic development of mouse forelimbs. Drug Chem Toxicol 35: 432-444, 2012

45. Zhu Y, Ren C, Wan X, Zhu Y, Zhu J, Zhou H and Zhang T: Gene expression of Hsp70, Hsp90 and Hsp110 families in normal palate and cleft palate during mouse embryogenesis. Toxicol Ind Health 29: 915-930, 2013.

46. Lavery LA, Partridge JR, Ramelot TA, Elnatan D, Kennedy MA and Agard DA: Structural asymmetry in the closed state of mitochondrial Hsp90 (TRAP1) supports a two-step ATP hydrolysis mechanism. Mol Cell 53: 330-343, 2014.

47. Dobbelstein M and Moll U: Targeting tumour-supportive cellular machineries in anticancer drug development. Nat Rev Drug Discov 13: 179-196, 2014. 